> La génomique du cancer du sein ouvre la voie à des traitements toujours plus personnalisés. Alors que le nombre de thérapies ciblées en développement augmente, les analyses à haut débit ont révélé l'hétérogénéité moléculaire du cancer du sein, identifié plusieurs sous-types moléculaires de la maladie très différents les uns des autres, un grand nombre d'altérations oncogéniques parfois très rares, et des signatures multigéniques prédictives de l'évolution clinique, dont certaines sont en cours de validation dans des essais cliniques prospectifs. Cette segmentation moléculaire du cancer en entités rares et la multitude de nouvelles molécules à tester (seules et en combinaison) conduisent à mettre en place des essais thérapeutiques basés sur le profil moléculaire des tumeurs afin d'orienter les patientes vers la molécule la plus adaptée. <

Avec 53000 nouveaux cas et 11500 décès cumulés pour l'année 2011 , le cancer du sein est le cancer le plus fréquent et le plus meurtrier chez la femme en France. Le dépistage de masse et l'amélioration des traitements, notamment systémiques (chimiothérapie [CT], hormonothérapie $[\mathrm{HT}]$, thérapies ciblées), ont fait diminuer la mortalité. Au niveau moléculaire, la maladie est caractérisée par l'accumulation et la combinaison de multiples anomalies génétiques et épigénétiques à l'origine de l'apparition de la tumeur, de l'instabilité génomique et de l'acquisition d'un phénotype invasif et résistant qui aboutira à la métastase et au décès. L'hétérogénéité clinique résultante, notamment évolutive, est mal appréhendée par les paramètres histocliniques pronostiques actuels, empêchant l'utilisation du traitement le plus adapté.

La prise en charge des patientes, basée initialement sur des facteurs histocliniques, se personnalise un peu plus grâce à la biologie. L'analyse biochimique des récepteurs hormonaux $(\mathrm{RH})$ des tumeurs avait ouvert la voie. Aujourd'hui, I'immunohistochimie (IHC), analysant l'expression des $\mathrm{RH}$ et du récepteur à activité tyrosine

\section{Génomique et recherche clinique en cancérologie mammaire}

\author{
François Bertucci $^{1,2,3}$, Daniel Birnbaum ${ }^{2}$
}

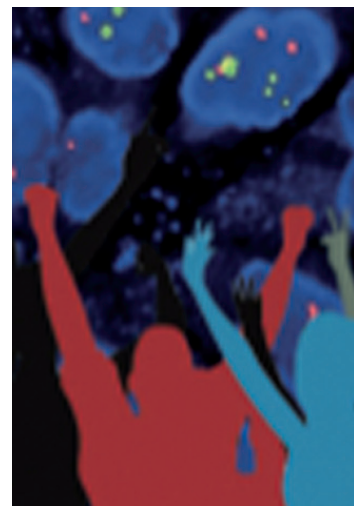

${ }^{1}$ Département d'oncologie médicale, Institut PaoliCalmettes, centre de recherche en cancérologie de Marseille, UMR891 Inserm ;

2 département d'oncologie moléculaire, Institut PaoliCalmettes, centre de recherche en cancérologie de Marseille, UMR891 Inserm, 232, boulevard Sainte-Marguerite, 13273 Marseille Cedex 9, France : ${ }^{3}$ université Aix-Marseille. bertuccif@marseille.fnclcc.fr

kinase HER2, I'hybridation in situ par fluorescence recherchant l'amplification du gène $H E R 2 / \varepsilon R B B 2$, et la recherche de mutations constitutionnelles de $B R C A 1$ et $B R C A 2$, sont utilisées en routine clinique et toutes ces molécules constituent autant de cibles thérapeutiques, pronostiques et prédictives de la réponse thérapeutique ( $\mathrm{RH}$ et H\&R2), ou de marqueurs de dépistage des formes héréditaires (mutations de $B R C A 1 / 2)$. Malgré ces progrès, l'évolution reste fatale chez 15 à $20 \%$ des patientes.

Un des enjeux majeurs de la recherche est le développement de molécules plus spécifiques ciblant une altération moléculaire clé de la tumeur, comme le fait le trastuzumab, anticorps monoclonal dirigé contre HER2, qui est surexprimé dans $20 \%$ des tumeurs. Parallèlement, alors que de nouvelles molécules prometteuses ne cessent de se développer, un bon nombre d'entre elles ont produit des résultats décevants dans les essais thérapeutiques, rendant cruciale l'identification de facteurs pronostiques et prédictifs de la réponse plus fiables. Dans ce contexte, une caractérisation moléculaire approfondie et globale des tumeurs est devenue indispensable. II y a une quinzaine d'années, l'arrivée de la génomique et ses techniques d'analyse moléculaire à haut débit ont révolutionné la recherche, permettant d'analyser le statut de milliers de gènes simultanément. La technique la plus utilisée a été celle des puces à ADN [1] pour l'analyse du transcriptome (ARN messagers), et, à un degré moindre, l'analyse du nombre de copies d'ADN (CGH-array) et des polymorphismes (SNP, single nucleotid polymorphisms). Le cancer du sein a été l'un des premiers cancers explorés par la génomique, dont sont issues aujourd'hui des applications en recherche clinique en France, mais déjà en routine dans d'autres pays. Cet article présente les résultats les plus prometteurs 
de la génomique en termes de débouchés cliniques, puis discute les répercussions sur la recherche clinique.

\section{Génomique et cancer du sein : des résultats prometteurs}

Ces études rétrospectives de cancers du sein ont permis d'appréhender pour la première fois l'hétérogénéité moléculaire de la maladie. II s'agit essentiellement d'analyses du transcriptome sur puces à ADN.

\section{Transcriptome et sous-types moléculaires}

En 2000, l'équipe américaine de C. Perou a défini, à partir d'un jeu intrinsèque de 500 gènes, cinq sous-types moléculaires (luminal $A$, luminal $B$, basal, HER2, normal-like) pertinents aux niveaux biologique et clinique [2, 3]. Ces sous-types sont liés au type cellulaire d'origine (luminal ou basal myoépithélial) et à des altérations moléculaires majeures de l'expression des RH et d'HER2 et de la prolifération. Ils se distinguent également par des différences épidémiologiques, histologiques et cliniques, notamment évolutives, en termes de réponse thérapeutique et de risque métastatique. Ces sous-types ont été observés dans différentes formes histocliniques, du cancer in situ [4] aux métastases [5] en passant par les formes inflammatoires [6]. Ils n'ont pas d'application pronostique directe pour l'instant pour plusieurs raisons: leur relative instabilité, de nouveaux sous-types, comme le sous-type claudin-low [7], étant identifiés au fur et à mesure que le nombre de tumeurs analysées augmente, le problème non résolu de l'applicabilité des puces en clinique (nécessité de tissu congelé), et l'absence de démonstration formelle de la valeur pronostique ajoutée. L'intérêt est conceptuel : le cancer du sein n'est plus reconnu comme une simple entité, mais comme cinq ou six maladies très distinctes ayant chacune des facteurs pronostiques et des cibles thérapeutiques différents, et devant donc être individualisées les unes des autres dans les essais cliniques. Au moins deux tests qui définissent ces soustypes sont déjà commercialisés : BluePrint ( 80 gènes, Agendia, PaysBas) et Breast BioClassifier (50 gènes, ARUP, États-Unis).

\section{Transcriptome et signatures prédictives de l'évolution clinique}

Ces signatures multigéniques concernent le risque de rechute métastatique et/ou la réponse thérapeutique $[8,9]$. Les signatures pronostiques ont été identifiées avec l'objectif de parvenir à une désescalade thérapeutique compte tenu du surtraitement par chimiothérapie adjuvante préconisé dans les formes localisées. II s'agit notamment des formes sans envahissement ganglionnaire $(\mathrm{N}-)$, où ce traitement, potentiellement toxique, est très souvent prescrit, bien qu'en réalité, chez plus de $60 \%$ des patientes, il ne soit pas nécessaire (cas surtraités devant bénéficier d'une désescalade thérapeutique) ; qu'il est nécessaire et efficace chez seulement $20 \%$ des patientes, et nécessaire mais inefficace à nouveau chez $20 \%$. Certaines signatures sont commercialisées. La signature Mammaprint (Agendia, Pays-Bas) inclut 70 gènes et a été validée par trois grandes séries rétrospectives chez des patientes N- [10-12]. Approuvée par la FDA (Food and drug administration) américaine, elle représente le premier test utilisant des puces à ADN commercialisé. Le test nécessite un échantillon tissulaire congelé. Le résultat rendu distingue un risque de rechute «faible » ou « haut ». La deuxième signature est le test Oncotype DX, commercialisé aux ÉtatsUnis par Genomic Health (Redwood City, CA). Elle comprend 16 gènes d'intérêt et cinq gènes de référence, dont les transcrits sont analysés en RT-PCR quantitative sur un échantillon de tumeur inclus en paraffine. Oncotype DX permet de classer les patientes $\mathrm{RH}+$ traitées par tamoxifène en trois classes: risque faible, intermédiaire ou élevé de rechute à 10 ans [13]. Oncotype est très utilisé aux États-Unis avec plus de 190000 tests réalisés depuis sa commercialisation, et, pour la première fois, Genomic Health a réalisé un bénéfice $(3,4$ millions de dollars) en 2010 . Toujours dans cette catégorie des tumeurs $\mathrm{N}-\mathrm{RH}+$, le ratio d'expression $\mathrm{HOXB13/}$ ILI7BR [14] est utilisé dans un test commercialisé aux États-Unis (kit Breast Cancer Gene Expression, Quest Diagnostics). Une autre signature, le Grade Genomic Index (GGI), comprend 97 gènes majoritairement impliqués dans la prolifération cellulaire [15]. II reclasse les grades histologiques II, de valeur pronostique incertaine et de faible reproductibilité, en grade génomique faible ou élevé, de bon et de mauvais pronostic respectivement. Le test est commercialisé depuis Juin 2008 (MapQuant DX, Ipsogen, France).

Des signatures prédictives de la réponse thérapeutique ont été rapportées après CT néoadjuvante à base d'anthracyclines et/ou de taxanes [8]. Une signature de 30 gènes développée par le MD Anderson Cancer Center (Texas, États-Unis) a été identifiée chez des patientes traitées par paclitaxel suivi de $\mathrm{FAC}^{1}$ [16]. Une signature de réponse aux anthracyclines (épirubicine seule) a été rapportée dans les tumeurs $\mathrm{RH}-$ [17]. Nous avons identifié sur une série de 468 patientes $\mathrm{N}$ + une signature prédictive de l'évolution clinique après CT adjuvante à base d'anthracycline sans taxane [18].

\section{Autres analyses moléculaires à haut débit}

Les autres niveaux moléculaires ( $A D N$, exome, miARN, protéome, épigénome, métabolome, etc.) analysés à haut débit n'ont pas encore atteint le degré d'avancement du transcriptome. Mais certains résultats sont déjà prometteurs. L’hybridation génomique comparative sur puces à ADN (array-CGH) définit en fonction de leur profil génomique global trois groupes de cancers du sein («simplex $»$, « complex», et «amplifier »), reflets de l'instabilité génomique. Ces profils pourraient aider l'évaluation de nouvelles molécules telles que les inhibiteurs de PARP (poly-ADP-ribose polymérase),

${ }^{1} 5$-Fluoro-uracile (F), cyclophosphamide (C) et une anthracycline (A) comme la doxorubicine ou l'épirubicine. 
particulièrement efficaces sur les tumeurs instables. Des signatures pronostiques ont aussi été développées $[19,20]$. Au niveau génique, de nombreuses altérations ont été révélées, mais souvent avec une récurrence faible (1-10\%). Les régions touchées sont souvent larges et multigéniques. Le tri des gènes altérés est en cours pour identifier les candidats, notamment en intégrant les données d'expression. Nous avons ainsi rapporté ZNF703 comme un gène candidat du sous-type luminal $B[21]$. Le séquençage de l'ADN est une autre approche prometteuse. Au niveau de l'ADN constitutionnel, bénéficiant du catalogue des variants génétiques humains (projet «1000 Génomes ») [22], les puces à SNP (single nucleotid polymorphisms) ont permis d'identifier dans le cadre des GWAS (genome-wide association studies) des SNP associés à une susceptibilité constitutionnelle à la maladie [23] ou à la survenue d'effets secondaires après traitement spécifique [24]. Actuellement ouverte en France, l'étude Signal, soutenue par l'Institut national du cancer, cherche à identifier, dans un prélèvement sanguin chez des femmes présentant un cancer du sein opéré et traité par trastuzumab en adjuvant, des déterminants génétiques associés à divers critères : résistance/sensibilité au trastuzumab, toxicité cardiaque après traitement adjuvant incluant le trastuzumab, sous-types moléculaires, et enfin risque de développer un cancer du sein. Au niveau somatique, des projets de séquençage complet du génome tumoral sont en place, comme le projet IGCC (international cancer genome consortium).

\section{Applications en recherche clinique}

La génomique permet donc d'analyser à l'échelle pangénomique les variations génétiques des patientes, l'instabilité génomique de leurs tumeurs ainsi que leurs profils d'expression génomique, de méthylation et de mutations. La génomique et/ou les résultats obtenus sont de plus en plus intégrés aux protocoles de recherche clinique qui représentent leur premier niveau d'application. Cette intégration a d'importantes répercussions sur les essais thérapeutiques.

\section{Plus d'essais cliniques}

La première conséquence est quantitative : le nombre d'essais à mettre en place augmente, qu'il s'agisse de nouvelles thérapies (ciblées ou non) ou de stratégies thérapeutiques plus anciennes. C'est une conséquence directe du nombre croissant de cibles moléculaires potentielles identifiées, mais également de l'hétérogénéité moléculaire du cancer du sein et sa segmentation en sous-types et autres sous-classes très différents les unes des autres. Toutefois, la mise en place d'essais basés sur le profil moléculaire des tumeurs devrait éviter cette escalade.

\section{Des essais cliniques différents \\ Incorporation de la génomique de façon rétrospective}

D'autres conséquences concernent le design des essais. La première est relativement simple. II s'agit de l'incorporation de la génomique dans l'essai thérapeutique en tant qu'outil à la recherche de déterminants moléculaires de la réponse à, ou de l'activité de, la drogue testée. L'idée est d'identifier, de manière rétrospective sur des échantillons tissulaires (tumeur et/ou tissu sain) du patient traité dans l'essai, des biomarqueurs d'activité pharmacodynamique (déphosphorylation par exemple pour un inhibiteur de kinase analysée sur des prélèvements réalisés séquentiellement avant, durant et après le traitement) et/ou des biomarqueurs de réponse tumorale, comme le statut des mutations de KRAS pour la réponse au cétuximab [25]. L'inclusion de la génomique est aujourd'hui quasiment systématique dans les essais thérapeutiques, étant donné son potentiel et l'importance des biomarqueurs dans le développement du médicament. L'organisation implique une logistique de collecte, stockage et transfert des échantillons, en sachant que les analyses moléculaires sont réalisées en fin d'essai, et non pas en temps réel. Cette intégration d'analyses génomiques soulève également les questions du consentement des patients, des surcoûts engendrés et de la propriété intellectuelle des échantillons et des données moléculaires générées.

\section{Essais cliniques basés sur la génomique}

Dans ces essais, l'inclusion des patientes va se faire en fonction des données moléculaires. On peut distinguer deux types d'essais dont l'objectif est différent:

- Dans le premier cas, l'essai a pour objectif de démontrer de façon prospective l'intérêt clinique de la signature génomique, phase ultime avant son application en routine. Les signatures pronostiques et prédictives (Mammaprint, Oncotype, signature à 30 gènes du MD Anderson, notre signature d'évolution clinique après CT adjuvante à base d'anthracycline, etc.) ne sont pas encore appliquées en routine en France. Leur valeur clinique ajoutée reste discutée par certains et les modalités de leur mise en route ne sont pas clairement identifiées. Mais surtout, puisque leur objectif est le plus souvent une désescalade thérapeutique par rapport aux standards histocliniques, le niveau de preuve requis avant leur acceptation est la démonstration, dans un essai randomisé prospectif, que le groupe de patients classé comme à faible risque a un pronostic en effet excellent ou ne bénéficie pas de la CT théoriquement indiquée. Plusieurs essais prospectifs sont en cours. La signature Mammaprint est en cours de validation dans l'essai européen MINDACT (Microarray in node-negative disease may avoid chemotherapy trial), qui a inclus 66000 patientes entre février 2007 et juillet 2011. L'objectif est de démontrer que l'utilisation de la signature, par rapport à celle du critère histoclinique Adjuvant! Online, permet de diminuer le nombre de patientes traitées par CT adjuvante en gardant une survie pourtant similaire [26]. De la même façon, le 
test Oncotype est testé dans l'essai américain TAILORx (Trial assigning individualized options for treatment $R x$ ) qui a inclus 10000 patientes $\mathrm{N}-\mathrm{RH}+$ entre mai 2006 et octobre 2010 [27]. La signature de 30 gènes du MD Anderson [16] a été validée dans un essai prospectif randomisé comparant ce protocole à l'administration de FAC sans taxane [28], sans toutefois apporter une information supplémentaire par rapport au prédicteur clinique. Elle est aujourd'hui en évaluation prospective dans l'essai français REMAGUS 04. Nous avons récemment clôturé l'essai SA02 (voir Encadré) basé sur notre signature pronostique après CT adjuvante [29]. II s'agit bien évidemment d'essais complexes en raison de la logistique de mise en place qui doit obéir à des impératifs de délais de rendu du résultat (4 à 6 semaines au maximum). L'analyse génomique se fait en temps réel puisque son résultat conditionne l'inclusion et le traitement. Le nombre de consentements peut être important. Dans le MINDACT, quatre consentements sont requis (envoi de la tumeur pour analyse spécifique, participation à l'essai, types de chimiothérapie et d'hormonothérapie). Les résultats de ces essais seront connus dans moins de 5 ans.

- Enfin, le deuxième type d'essai basé sur la génomique est encore plus proche de la médecine personnalisée. II concerne les stades métastatiques dans lesquels les nouvelles drogues sont testées : il s'agit de définir le profil génomique de la tumeur afin d'orienter l'inclusion dans l'essai thérapeutique le plus adapté. $\varepsilon$ n effet, la génomique a identifié des altérations d'un nombre important de gènes et protéines dans le cancer du sein, mais le plus souvent avec une fréquence faible, infé-

\section{Essai clinique SAO2}

Le bénéfice de la chimiothérapie (CT) adjuvante (post-opératoire) est démontré en termes de survie dans le cancer du sein localisé opéré. Pour les patientes avec envahissement ganglionnaire axillaire $(\mathrm{N}+)$, cette CT est aujourd'hui basée sur un protocole à base d'anthracycline et taxane. Toutefois, le bénéfice lié à l'addition des taxanes concerne une population relativement limitée au prix d'un coût morbide et financier élevé. Identifier les patientes bénéficiant ou pas d'un protocole à base d'anthracycline sans taxane est crucial pour améliorer l'index thérapeutique. Dans ce but, nous avions identifié sur puces à ADN sur une série rétrospective de 468 patientes $N+$ une signature multigénique prédictive de la rechute métastatique après CT adjuvante à base d'anthracycline sans taxane [18]. Afin de valider l'évolution favorable de la population prédite de «bon pronostic » par cette signature, nous avons lancé l'étude SA02, cohorte nationale prospective de CT adjuvante basée sur notre signature. Les patientes $N+$ opérées dont la signature génomique réalisée en temps réel prédisait un «bon pronostic » se voyaient proposer un protocole à base d'anthracycline sans taxane ( 6 cures de $\mathrm{FEC100)}$ ), au lieu du protocole standard ( 3 FEC100 suivies de 3 cures de docetaxel). Au total, 175 patientes ont été incluses dans l'étude (fin des inclusions, mai 2010). L'objectif primaire était la survie sans métastase à 5 ans. Les objectifs secondaires incluaient entre autres la survie globale à 5 ans, la faisabilité de la génomique en temps réel et l'évaluation socio-économique et psychologique de l'approche. L'analyse préliminaire des résultats [29] a révélé un taux d'échec de la génomique de $34 \%$, l'absence de rechute métastatique après un suivi médian de 13 mois, et une très bonne acceptabilité par les patientes. rieure à $10 \%$, voire $5 \%$. De multiples anomalies peuvent être présentes dans la même tumeur, ce qui lui confère son agressivité. Parallèlement, plusieurs centaines de thérapies ciblées sont en développement en cancérologie, ce qui générera plusieurs milliers d'associations thérapeutiques qu'il faudra également évaluer. Or, ces drogues et/ou ces associations ne seront potentiellement efficaces que chez le petit nombre de patientes dont la cible est altérée dans la tumeur. Ce contexte rend incongrue, et surtout non souhaitable, la stratégie actuelle qui consiste à réaliser un essai thérapeutique dans la population tout-venant, puis à chercher ensuite à identifier le biomarqueur prédictif de façon rétrospective. Un criblage moléculaire initial à la recherche des cibles doit déboucher sur des effectifs plus réduits, des essais plus rapides et moins coûteux, et surtout l'administration d'un traitement a priori plus efficace. Le criblage peut rechercher une anomalie «large » telle que l'instabilité du génome (anti-PARP 1 et 2), ou plus précise comme l'activation d'une voie de signalisation (signature des voies RAS ou MYC activées [30]), ou une altération génique au niveau de I'ADN (mutation EGFR [epidermal growth factor receptor], translocation $E M L 4$ / ALK [anaplastic lymphoma kinase], etc.). L'intérêt de cette approche a été récemment démontré dans une étude [31] : l'expression de 61 gènes/protéines cibles de thérapies existantes y était analysée par IHC et puces à ADN à partir d'un échantillon de tumeur chez 86 patients atteints d'un cancer métastatique réfractaire. Une cible thérapeutique était détectée chez 84 patients, dont 66 recevaient alors le traitement correspondant. Pour 18 d'entre eux, le temps de survie sans progression avec ce traitement était supérieur de plus de 1,3 fois au temps de survie sans progression observé avec le traitement précédent. Un essai proche de celui-ci, SAFIR01, promu par Unicancer, a débuté en France dans le cancer du sein métastatique. Une fois le consentement signé par les patients, un échantillon est prélevé au niveau d'un site tumoral métastatique. L'analyse du prélèvement comprend la recherche d'amplifications en CGH-array et de mutations des voies mTOR (PI3K, PTEN, AKT). L'analyse bioinformatique détermine ensuite la présence d'une cible moléculaire permettant d'orienter la patiente vers un essai précoce de thérapies ciblées [32]. En enrichissant la population en patients présentant l'anomalie moléculaire cible, cette approche doit accélérer le développement précoce du médicament. Mais elle requiert (1) un test robuste et facilement applicable en clinique, permettant d'évaluer un grand nombre d'altérations moléculaires potentielles susceptibles d'être ciblées et reconnues dans des modèles précliniques comme prédictives de l'efficacité, et (2) surtout un algorithme 
bioinformatique capable de définir la ou les cibles d'intérêt dans une tumeur donnée [33].

\section{Conclusion}

La génomique représente un tournant pour la recherche biomédicale. En cancérologie mammaire, elle devrait ouvrir la voie vers des traitements de plus en plus personnalisés. Des évolutions technologiques en cours vont permettre d'en élargir l'application à des échantillons cliniques autrefois inutilisables (fixation en paraffine, cellules tumorales circulantes), mais également d'augmenter la résolution de la détection d'altérations (puces SNP à très haute résolution ou séquençage de nouvelle génération). Des défis persistent avant que cette médecine moléculaire ne devienne routine ; ils concernent notamment des questions logistiques, financières (coût, remboursement des drogues et des tests moléculaires), réglementaires (consentements, brevets, agrément), mais également des questions d'acceptation et de compréhension par les patientes. $\diamond$

\section{SUMMARY}

Genomics and clinical research for breast cancer

Genomics of breast cancer is paving the way towards more and more tailored treatments. The number of molecularly targeted therapies under development is increasing. In parallel, the high-throughput analyses revealed the molecular heterogeneity of disease, and identified several very different molecular subtypes, numerous and sometimes very scarce molecular alterations, and multigenic signatures predictive for clinical outcome, some of which are being tested in prospective clinical trials. This molecular segmentation of breast cancer and the multitude of new drugs to be tested (alone and in combination) lead to develop clinical trials based on the molecular profile of tumors to guide the patient towards the most suitable drug. $\diamond$

\section{CONFLIT D'INTÉRÊTS}

Les auteurs déclarent n'avoir aucun conflit d'intérêts concernant les données publiées dans cet article.

\section{RéFÉRENCES}

1. Bertucci F, Houlgatte R, Nguyen C, et al. Gene expression profiling of cancer by use of DNA arrays: how far from the clinic? Lancet Oncol $2001 ; 2: 674-82$.

2. Perou CM, Sorlie T, Eisen MB, et al. Molecular portraits of human breast tumours. Nature 2000 ; $406: 747-52$.

3. Sorlie T, Perou CM, Tibshirani R, et al. Gene expression patterns of breast carcinomas distinguish tumor subclasses with clinical implications. Proc Natl Acad Sci USA 2001 ; 98 : 10869-74.

4. Yu K, Lee CH, Tan PH, Tan P. Conservation of breast cancer molecular subtypes and transcriptional patterns of tumor progression across distinct ethnic populations. Clin Cancer Res $2004 ; 10$ : 5508-17.

5. Weigelt B, Hu Z, He X, et al. Molecular portraits and 70-gene prognosis signature are preserved throughout the metastatic process of breast cancer. Cancer Res $2005 ; 65: 9155-8$.

6. Bertucci F, Finetti $P$, Rougemont J, et al. Gene expression profiling identifies molecular subtypes of inflammatory breast cancer. Cancer Res $2005 ; 65: 2170-8$.

7. Prat A, Parker JS, Karginova 0 , et al. Phenotypic and molecular characterization of the claudinlow intrinsic subtype of breast cancer. Breast Cancer Res $2010 ; 12:$ R68.

8. Sotiriou C, Pusztai L. Gene-expression signatures in breast cancer. $N$ EnglJ Med 2009 ; $360: 790-800$.

9. Bertucci F, Finetti P, Cervera N, Birnbaum D. Classification pronostique du cancer du sein et profils d'expression génique sur puces à ADN. Med Sci (Paris) 2008 ; 24 : 599-606.
10. van 't Veer LJ, Dai H, van de Vijver MJ, et al. Gene expression profiling predicts clinical outcome of breast cancer. Nature $2002 ; 415: 530-6$.

11. van de Vijver MJ, He YD, van't Veer LJ, et al. A gene-expression signature as a predictor of survival in breast cancer. $N$ Engl J Med 2002 ; 347 : 1999-2009.

12. Buyse M, Loi S, van't Veer L, et al. Validation and clinical utility of a 70 -gene prognostic signature for women with node-negative breast cancer. J Natl Cancer Inst 2006 ; 98 : 1183-92.

13. Paik S, Shak S, Tang G, et al. A multigene assay to predict recurrence of tamoxifen-treated, node-negative breast cancer. N EnglJ Med 2004 ; $351: 2817-26$

14. Ma XJ, Wang Z, Ryan PD, et al. A two-gene expression ratio predicts clinical outcome in breast cancer patients treated with tamoxifen. Cancer Cell $2004 ; 5: 607-16$.

15. Sotiriou C, Wirapati P, Loi S, et al. Gene expression profiling in breast cancer: understanding the molecular basis of histologic grade to improve prognosis. J Natl Cancer Inst $2006 ; 98: 262-72$.

16. Hess KR, Anderson K, Symmans WF, et al. Pharmacogenomic predictor of sensitivity to preoperative chemotherapy with paclitaxel and fluorouracil, doxorubicin, and cyclophosphamide in breast cancer. J Clin Oncol 2006 ; $24: 4236-44$

17. Desmedt C, Di Leo A, de Azambuja $\varepsilon$, et al. Multifactorial approach to predicting resistance to anthracyclines. J Clin Oncol 2011 ; 29 : 1578-86.

18. Bertucci F, Borie N, Roche H, et al. Gene expression profile predicts outcome after anthracycline-based adjuvant chemotherapy in early breast cancer. Breast Cancer Res Treat $2011 ; 127$ : 363-73.

19. Gravier $\varepsilon$, Pierron G, Vincent-Salomon A, et al. A prognostic DNA signature for T1T2 node-negative breast cancer patients. Genes Chromosomes Cancer $2010 ; 49: 1125-34$.

20. Zhang $Y$, Martens JW, Yu JX, et al. Copy number alterations that predict metastatic capability of human breast cancer. Cancer Res 2009; $69: 3795-801$.

21. Sircoulomb F, Nicolas N, Ferrari A, et al. ZNF703 gene amplification at $8 \mathrm{p} 12$ specifies luminal B breast cancer. EMBO Mol Med 2011 ; 3 : 153-66.

22. Mills RE, Walter $K$, Stewart $C$, et al. Mapping copy number variation by population-scale genome sequencing. Nature $2011 ; 470: 59-65$.

23. Thomas $G$, Jacobs $K B$, Kraft $P$, et al. A multistage genome-wide association study in breast cancer identifies two new risk alleles at 1 p 11.2 and 14q24.1 (RAD51L1). Nat Genet 2009; $41: 579-84$.

24. Ingle JN, Schaid DJ, Goss PE, et al. Genome-wide associations and functional genomic studies of musculoskeletal adverse events in women receiving aromatase inhibitors. J Clin Oncol $2010 ; 28: 4674-82$.

25. Lievre A, Bachet JB, Le Corre D, et al. KRAS mutation status is predictive of response to cetuximab therapy in colorectal cancer. Cancer Res 2006 ; $66: 3992-5$.

26. Cardoso F, Van't Veer L, Rutgers $\varepsilon$, et al. Clinical application of the 70 -gene profile: the MINDACT trial. J Clin Oncol 2008 ; 26 : 729-35.

27. Sparano JA, Paik S. Development of the 21 -gene assay and its application in clinical practice and clinical trials. J Clin Oncol $2008 ; 26: 721-8$.

28. Tabchy A, Valero V, Vidaurre T, et al. Evaluation of a 30 -gene paclitaxel, fluorouracil, doxorubicin, and cyclophosphamide chemotherapy response predictor in a multicenter randomized trial in breast cancer. Clin Cancer Res $2010 ; 16: 5351-61$.

29. Bertucci F, Ferrero JM, Bachelot T, et al. SA02 trial: a genomics-based prospective study of adjuvant chemotherapy in node-positive early breast cancer with good-prognosis signature. San Antonio, TX: Breast Cancer Symposium, 2009.

30. Bild AH, Parker JS, Gustafson AM, et al. An integration of complementary strategies for gene-expression analysis to reveal novel therapeutic opportunities for breast cancer. Breast Cancer Res 2009 ; 11 : R55.

31. Von Hoff DD, Stephenson JJ Jr, Rosen P, et al. Pilot study using molecular profiling of patients' tumors to find potential targets and select treatments for their refractory cancers. J Clin Oncol $2010 ; 28: 4877-83$.

32. Andre F, Delaloge S, Soria JC. Biology-driven phase II trials: what is the optimal model for molecular selection? J Clin Oncol 2011; 29 : 1236-8.

33. Tursz T, Andre F, Lazar V, LaCroix L, Soria JC. Implications of personalized medicine-perspective from a cancer center. Nat Rev Clin Oncol 2011 ; $8: 177-83$
TIRÉS À PART

F. Bertucci 